\title{
Introduction to the Knowledge Flows, Sharing, Transfer, and Exchange Minitrack
}

\author{
Murray E. Jennex \\ San Diego State University \\ mjennex@mail.sdsu.edu
}

\author{
Gang Peng \\ California State University Fullerton \\ gpeng@fullerton.edu
}

\author{
Liana Razmerita \\ Copenhagen Business School \\ lra.msc@cbs.dk
}

Knowledge management is driven by the flow of knowledge in an organization. Most practitioners and researchers recognize that knowledge flows are extremely important, especially in the current age where organizations have to learn and innovate continually in order to remain competitive. Given that knowledge is distributed unevenly through the enterprise, rapid and reliable knowledge flows are essential for organizations' sustainable competitive advantage.

Knowledge flows across people, organizations, places and times. Knowledge flows are dynamic as they involve creation, sharing, and application. And in order to harness the competitive power of this dynamic phenomenon, it is imperative to understand and manage effectively the associated knowledgeflow processes.

This minitrack examines the nature and role of knowledge flows (e.g., knowledge transfer, sharing and exchange) in the organizational context. The Call for Papers welcomed theoretical and empirical pieces addressing technical, managerial, behavioral, organizational, and economic perspectives on knowledge flows.

Seventeen submissions were received and seven were accepted for presentation in two sessions. A brief summaries of the papers are presented here.

Understanding the Emotional and Informational Influence on Product Knowledge Contribution through Quantitative Content Analysis by Xiaolei Wang, Terence T. Ow, and Yuqiang Feng is our best paper nomination. This paper attempts to elaborate the influence of emotional support and informational support on product knowledge contribution of customers in a firm-hosted online community. It uses quantitative content analysis including product feature extraction and sentiment analysis, from 2318 users of an online community.

Barriers of Knowledge transfer between geographically distributed teams in ICT product development by Aicha Togola, Safa Ahmed, and Taline Jadaan focused on identifying the main barriers that globally distributed teams face in conducting KT and how these barriers are unraveled. It is based on an interpretative case study of a product development unit at a R\&D department in a Telecom.

Knowledge Sharing Practices, Intellectual Capital and Organizational Performance by Mujid M. Attar, Kyeong Kang, and Osama Sohaib explores whether knowledge sharing practices (types, approaches, and process) and intellectual capital affect organizational operational performance.

Legitimate Peripheral Participation and Value Creation in Online Knowledge Sharing Communities by Hani Safadi and Nicholas Berente, theorizes about how legitimate peripheral participation of new online knowledge sharing community members can drive different types of contributions and potentially generate value for the community.

Knowledge Adoption: A New Perspective and the Influence of Knowledge Characteristics by Nan Wang provides a new perspective of knowledge adoption that includes two processes (i.e., awareness and evaluation) and three possible adoption outcomes (strong adoption, weak adoption, and faint adoption).

Method for Eliciting and Analyzing Business Processes Based on Storytelling Theory by Pedro Antunes, Jose A. Pino, and Mary Tate suggests that storytelling theory can be used to analyze business processes in two behavioral dimensions: model and context, which concern predefined and improvised behavior.

Community by Design: Prioritizing the Factors that Drive Knowledge Use in Online Question \& Answers Platforms by Babajide James Osatuyi and Katia Passerini investigates factors that influence the use of knowledge in an online question and answer platform (OQA). It focuses on three levels including informational, individual, and community, and reviews interactions across each level.

The minitrack co-chairs want to thank authors and reviewers for their work in making this minitrack a success and we look forward to continue building this research community in the coming years. 